\title{
Exploring the Small Scale Structure of N103B
}

\author{
Karen T. Lewis ${ }^{1}$, David N. Burrows ${ }^{1}$, John A. Nousek ${ }^{1}$, \\ Gordon P. Garmire ${ }^{1}$, Patrick Slane ${ }^{2}$, John P. Hughes ${ }^{3}$ \\ 1 The Pennsylvania State University, 525 Davey Laboratory, University Park, PA 16801 \\ ${ }^{2}$ Harvard-Smithsonian Center for Astrophysics, 60 Garden Street, Cambridge, MA, 02138 \\ ${ }^{3}$ Department of Physics and Astronomy, Rutgers University, \\ 136 Frelinghuysen Road, Piscataway, NJ 08854-8019
}

\begin{abstract}
We present the preliminary results of a 40.8 ks Chandra ACIS observation of the young supernova remnant (SNR) N103B located in the Large Magellanic Cloud. The image reveals structure at the sub-arcsecond level, including several bright knots and filamentary structures. The remnant has the characteristic spectrum of a Type Ia SNR, containing strong lines of Fe, He-and H-like Si and S, Ar, and Ca. Narrow band images reveal non-uniformities in the remnant.
\end{abstract}

\section{INTRODUCTION}

N103B, one of the brightest X-ray and radio sources in the Large Magellanic Cloud (LMC), is a young, compact supernova remnant (SNR). The remnant, located on the north-eastern edge of the HII region N103, is $\sim 40 \mathrm{kpc}$ from the young star cluster NGC 1850. [1] The radio and X-ray morphologies are strikingly similar; the western edge (near the HII region) shows considerable structure and is $\sim 3$ times brighter than the eastern edge. [1,2] In both bands, the emission arises from a region $7 \mathrm{pc}$ in diameter ( $\mathrm{d}=50 \mathrm{kpc}$ to $\mathrm{LMC}$ assumed). Due to its proximity to a star forming region, it was originally suspected that N103B was the result of the core collapse of a massive object. However, the ASCA spectrum shows no evidence for $\mathrm{K}$-shell emission from $\mathrm{O}$, Ne or $\mathrm{Mg}$ while $\mathrm{Si}, \mathrm{S}, \mathrm{Ar}, \mathrm{Ca}$, and Fe features are strong, indicating that the remnant is more likely the result of a Type Ia SN explosion. [3]

\section{OBSERVATION AND DATA CLEANING PROCEDURE}

N103B was observed for 40.8 ks with ACIS S-3, one of the back-illuminated CCDs in the ACIS-S array. We found that $\sim 35 \%$ of the frames had a high background count rate and that during these times, the spectrum of the background changed 
significantly. These frames were removed from the data set. The standard ASCA grade filter (g 02346) was used.

\section{ANALYSIS AND RESULTS}

Our observation of N103B has confirmed many earlier results. The Chandra X-ray and the Australian Telescope Compact Array (ATCA) radio images [1] are quite similar (Figure 1). Since the radio and X-ray flux is depressed in the eastern half of the remnant we conclude that this intensity difference is due to a real density variation, as opposed to an absorption effect. While the bright radio knots are in the general vicinity of the X-ray knots, they do not overlap. Further investigation is required to determine whether there is a relative rotation between the two images, which may explain the offset. The spectrum of N103B as a whole (Figure 2) confirms the earlier ASCA results. We see no evidence for $\mathrm{O}$ or $\mathrm{Ne}$ in the spectrum, although a $\mathrm{Mg}$ line is present.

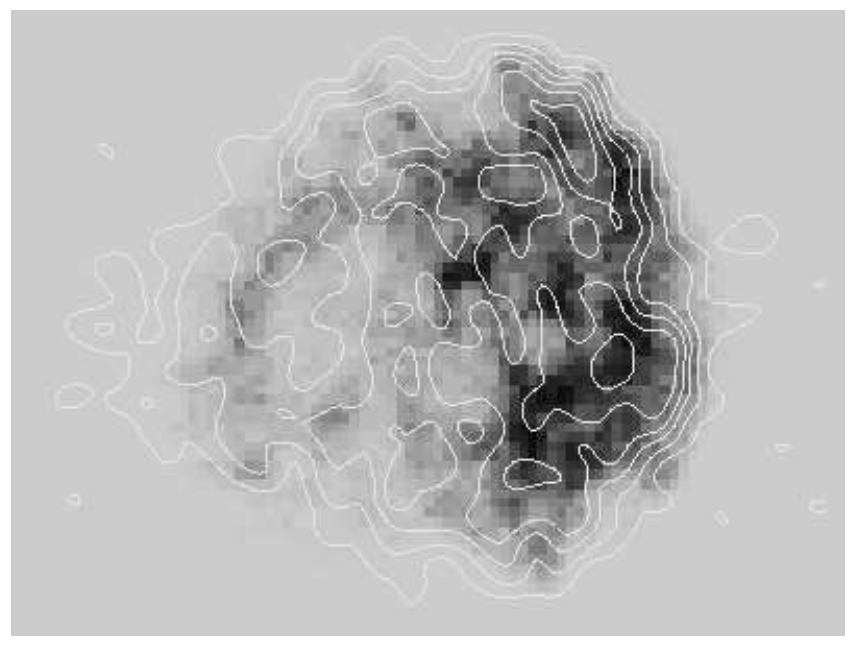

FIGURE 1. Plot of X-ray image with $2.5 \mathrm{~cm}$ ATCA contours [1]. Contours are 1.7, 1.3, 0.9, $0.6,0.3$, and $0.1 \mathrm{mJy} /$ beam. The spatial resolution of the radio data is 1.2 ", which is slightly larger than the Chandra's.

Continuum subtracted (CS) and equivalent width (EW) images show significant variation throughout the remnant. The images for He-like Si are shown in Figure 3. The interval 1700-1865 eV was used to create the He-like Si line image. To estimate the continuum, we performed a linear interpolation between images in the 1515$1680 \mathrm{eV}$ and 2050-2215 eV bands, which are free of any significant lines. All images were smoothed with a 3 pixel (1.5") boxcar to reduce the number of pixels with no counts. We were concerned that the CS and EW images were unreliable, as each pixel contained only a few counts at most, so the CS and EW images were collapsed into radial and azimuthal dimensions by binning the line and continuum images into rings and sectors. The CS and EW flux were calculated for each ring and sector 


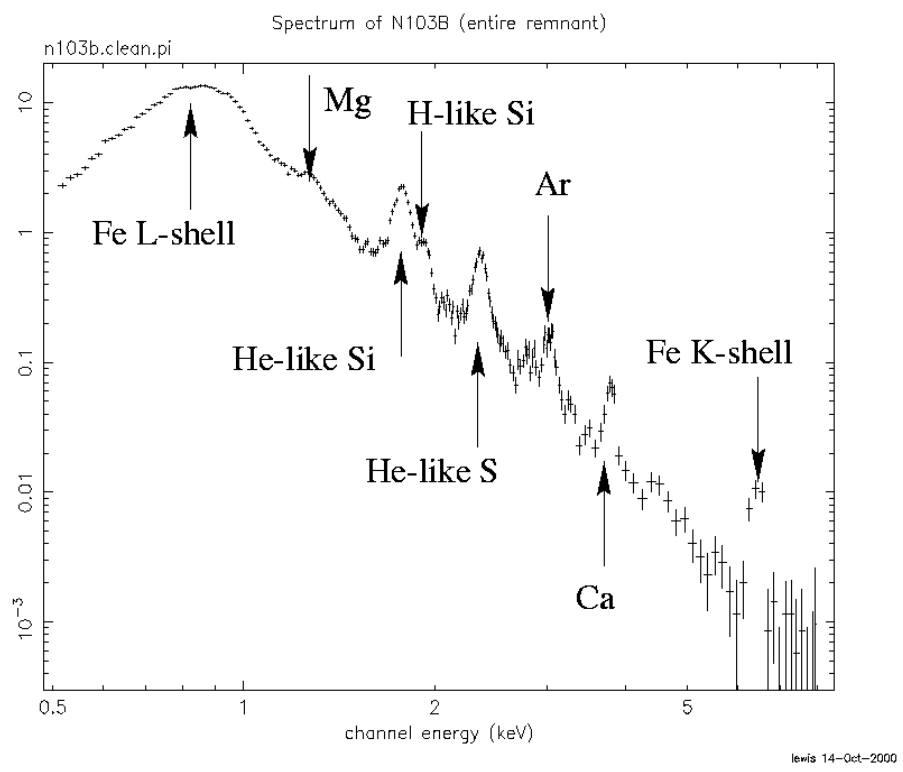

FIGURE 2. Spectrum of N103B as a whole. The data are plotted so that each data point has a 6 sigma significance with a maximum bin size of $\sim 65 \mathrm{eV}$

as shown in bottom of Figure 3. The radial and azimuthal plots confirm what is seen in the images. The emission from He-like $\mathrm{Si}$ is greatest along the western edge, where the entire remnant is bright. However, as shown by the EW images and plots, it is only in the outer rim of the remnant that the He-like Si emission drastically dominates over the continuum emission. Throughout the remainder of the remnant, the ratio of line to continuum is relatively flat. We have not yet determined whether the change in emissivity is due to a difference in abundance, temperature, ionization or a combination of these.

\section{CONCLUSIONS}

Preliminary analysis of this Chandra observation reveal that N103B shows significant structure at the arcsecond level. The variations in X-ray flux are consistent with those in radio and are due to real density variation within the remnant. The spectrum of the remnant is that of a typical Type Ia SNR, however future spatially resolved spectroscopy will allow for a better classification. Finally, there are dramatic spatial variations in the emissivity of He-like $\mathrm{Si}$ in particular. The radial variation in the equivalent width of the remnant is particularly intriguing. Detailed spectral analysis of concentric rings will enable us to determine the source of these and other similar variations. 

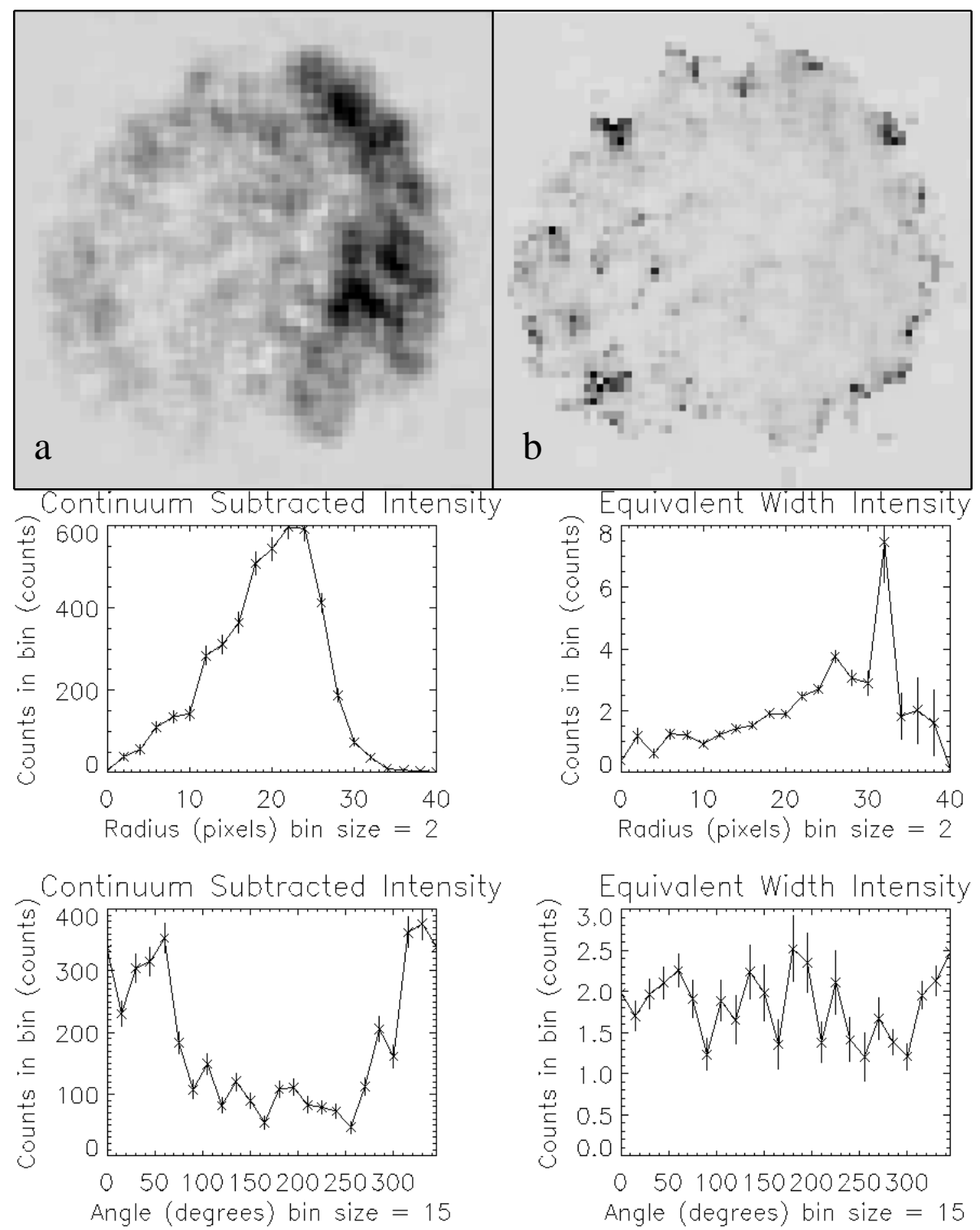

FIGURE 3. Plot of He-like Si continuum subtracted (a) and equivalent width images (b). Below are radial and azimuthal plots. 2 pixels $=1 " \sim 0.2 \mathrm{pc}$

\section{REFERENCES}

1. Dickel, J. and Milne, D. 1995, AJ, 109, 1

2. Mathewson, D.S., Ford, V.L., Dopita, M.A., Tuohy, I.R., Long, K.S., \& Helfand, D.J. 1983, ApJS, 51,345

3. Hughes, J.P, Hayashi, I., Helfand, D., Hwang, U., Itoh, M., Kirshner, R., Koyama, K., Markert, T., Tsunemi, H., \& Woo, J., 1995 ApJL, 444, L81 